\title{
Original article (short paper) \\ Carbohydrate mouth rinse reduces rating of perceived exertion but does not affect performance and energy systems contribution during a high-intensity exercise
}

\author{
Victor José Bastos-Silva \\ Gustavo Gomes de Araujo \\ Sérgio Victor dos Santos Franco \\ Alan de Albuquerque Melo \\ Universidade Federal de Alagoas, UFAL, Maceió, AL, Brazil \\ Sara Kely Learsi \\ Universidade Federal de Pernambuco, UFPE, Recife, PE, Brazil \\ Adriano Eduardo Lima-Silva \\ Universidade Tecnológica Federal do Paraná, UTFPR, Curitiba, PR, Brazil \\ Romulo Bertuzzi \\ Universidade de São Paulo, USP, São Paulo, SP, Brazil
}

\begin{abstract}
Aim: The study aimed to verify the effect of carbohydrate (CHO) mouth rinse on time to exhaustion, energy systems contribution and rating of perceived exertion (RPE) during a high-intensity exercise. Methods: Fourteen men performed an incremental exercise test to determine their maximal oxygen uptake and peak power output (PPO) and two time-to-exhaustion tests at $110 \%$ of PPO. Participants rinsed their mouth with $25 \mathrm{ml}$ of $6.4 \%$ of CHO or placebo (PLA) solution immediately before the time-to-exhaustion test, using a crossover design. The contribution of the energy systems was calculated using the free software GEDAE-LaB®. Results: Time to exhaustion was similar between the conditions (CHO:174.3 $\pm 42.8 \mathrm{~s}$; PLA:166.7 $\pm 26.3 \mathrm{~s} ; \mathrm{p}=0.33$ ). In addition, there was no difference between the $\mathrm{CHO}$ and PLA condition for aerobic (CHO:135.1 $\pm 41.2 \mathrm{~kJ}$ and PLA:129.8 $\pm 35.3 \mathrm{~kJ}, \mathrm{p}=0.34)$, anaerobic lactic (CHO:57.6 $\pm 17.1 \mathrm{~kJ}$ and PLA:53.4 $\pm 15.1 \mathrm{~kJ}, \mathrm{p}=0.10$ ), and anaerobic alactic (CHO:10.4 $\pm 8.4 \mathrm{~kJ}$ and PLA:13.2 $\pm 9.2 \mathrm{~kJ}, \mathrm{p}=0.37$ ) contribution. Consequently, total energy expenditure was similar between conditions (CHO:203.2 $\pm 46.4 \mathrm{~kJ}$ and PLA:196.5 $\pm 45.2 \mathrm{~kJ}$, $\mathrm{p}=0.15$ ). However, $\mathrm{CHO}$ mouth rinse reduced the RPE at the moment of exhaustion (CHO:18.2 \pm 1.0 units and PLA:19.1 \pm 1.1 units; $\mathrm{p}=0.02$ ). Conclusion: $\mathrm{CHO}$ mouth rinse neither increased time to exhaustion nor altered energy systems contribution during a high-intensity exercise, but reduced the perceived effort at the exhaustion.
\end{abstract}

Keywords: ergogenic aid; maltodextrin; mouthwash; supramaximal exercise; supplementation.

\section{Introduction}

Exogenous carbohydrate $(\mathrm{CHO})$ is a largely recognized nutritional supplement able to enhance exercise endurance since it provides "extra" CHO to oxidation when muscle glycogen becomes depleted ${ }^{1,2}$. However, during high-intensity exercises, the muscle glycogen is not fully emptied and $\mathrm{CHO}$ availability may not be a limiting factor for exercise performance ${ }^{2,3}$. Rather, it seems that $\mathrm{CHO}$ mouth rinse might be a promising strategy for improving performance during high-intensity exercise ${ }^{4}$. In fact, some studies have shown the efficacy of $\mathrm{CHO}$ mouth rinse before high-intensity effort ${ }^{5,6,7}$.

One possible explanation for improving the performance during high-intensity exercise with $\mathrm{CHO}$ mouth rinse is that the contact of $\mathrm{CHO}$ with the oral $\mathrm{CHO}$ receptors may trigger an afferent signal to the central nervous system ${ }^{8,9}$, which would be associated with a facilitation of motor output ${ }^{10}$. The $\mathrm{CHO}$ mouth rinse seems to stimulate reward-associated areas in the sensorimotor cortex such as insula/frontal operculum, orbitofrontal cortex and striatum ${ }^{8,9}$, which might be integrated with descending motor outputs and neuromuscular activation. Interestingly, activation of reward areas in the sensory cortex is also associated with an increased power output ${ }^{8,11}$ or longer time until exhaustion ${ }^{12}$ for the same rating of perceived exertion (RPE). In addition, due to the facilitation of motor output, there may be a greater recruitment of motor units, which may modify the energy profile during exercise.

The quantification of the energy contribution during the exercise is a key point for the development of strategies to improve performance ${ }^{13}$. However, there are no studies investigating the effects of $\mathrm{CHO}$ mouth rinse on energy systems contribution during high-intensity exercise. Thus, the purpose of the present study was to evaluate the influence of $\mathrm{CHO}$ mouth rinse on performance, energy systems contribution and RPE during a high-intensity exercise. It was hypothesized that $\mathrm{CHO}$ mouth rinse will improving performance and altering the contribution of energy systems during a high-intensity exercise. 


\section{Methods}

\section{Participants}

Fourteen healthy and physically active men (age: $23 \pm 2$ years; body mass: $74.1 \pm 11.4 \mathrm{~kg}$; height: $170.0 \pm 1.0 \mathrm{~cm}$; body fat: $14.6 \pm 6.7 \%$; $\left.\mathrm{VO}_{2 \max }: 40.0 \pm 7.4 \mathrm{ml} \cdot \mathrm{kg}^{-1} \cdot \mathrm{min}^{-1}\right)$, who performed a minimum of 150 min of physical activity per week, were recruited. Participants were provided with information regarding experimental risks and signed an informed consent form before starting the experiments. The study procedures were conducted in accordance with the Declaration of Helsinki (2008) and were approved by the local ethics committee (protocol number: 16573413.8.0000.5013).

\section{Experimental Design}

This study was conducted in a crossover, randomized, counterbalanced, and single-blind design. Participants performed three experimental sessions, separated by at least $72 \mathrm{~h}$. During the first visit, anthropometric parameters were obtained and a maximal incremental exercise test was performed to determine maximal oxygen uptake $\left(\mathrm{O}_{2 \max }\right)$ and the peak power output (PPO). In the second and third visits, a high-intensity exercise was performed at $110 \%$ PPO until voluntary exhaustion, which was preceded by either $\mathrm{CHO}$ or placebo (PLA). Mouth rinse was performed $10 \mathrm{~s}$ before the test. All tests were performed at the same time of the day, $2 \mathrm{~h}$ after the last meal ${ }^{14}$. Participants were instructed to replicate their food intake in the $24 \mathrm{~h}$ prior each experimental trial. Compliance with the diet recommendations was checked before each test by dietary recall. The participants were asked to refrain from exhaustive exercise as well as alcohol and caffeine ingestion $48 \mathrm{~h}$ prior to each experimental trial.

\section{Anthropometry and maximal incremental exercise test}

Firstly, body weight, height and skinfolds (chest, abdominal and thigh) were measured. Body density was estimated using the generalized equation of Jackson and Pollock ${ }^{15}$, and converted to body fat percentage using the equation of Siri ${ }^{16}$.

Then, a maximal incremental exercise test was carried out on an electromagnetically braked cycle ergometer (Ergo Fit 167, Ergo-FitGmbH \& Co., Pirmasens, Germany). The seat height was adjusted for each participant, allowing near full leg extension during each pedal revolution. The seat height was noted and reproduced in all subsequent experimental sessions. After a 3-min, warm-up at $30 \mathrm{~W}$, the power output was increased $30 \mathrm{~W}$ $\mathrm{min}^{-1}$ maintaining pedal cadence between 60 and $70 \mathrm{rpm}$ until voluntary exhaustion, which was defined as the incapacity to maintain a minimum pedal cadence of $60 \mathrm{rpm}$ for more than five consecutive seconds. The participants received strong verbal encouragement to continue as long as possible.

Oxygen uptake $\left(\mathrm{O}_{2}\right)$ was measured breath-by-breath throughout the test using a gas analyzer (Quark, Cosmed, Rome, Italy) and averaged over $30 \mathrm{~s}$ intervals. The calibration of the gas analyzer was performed according to manufacturer specifications using ambient air, a gas containing $20.9 \%$ of $\mathrm{O}_{2}$ and $5 \%$ of $\mathrm{CO}_{2}$, and a $3-\mathrm{L}$ syringe. $\mathrm{O}_{2 \max }$ was determined when two or more of the following criteria were met: an increase in $\mathrm{O}_{2}$ of less than $2.1 \mathrm{ml} \cdot \mathrm{kg}^{-1} \cdot \mathrm{min}^{-1}$ on two consecutive stages, a respiratory exchange ratio greater than 1.1 and to reach $\pm 10 \mathrm{bpm}$ of the maximal age-predicted heart rate ${ }^{17}$. The highest power output reached during the trial was considered as PPO.

\section{High-intensity exercise}

The high-intensity exercise was performed at $110 \%$ of PPO. This intensity was chosen based on Weber and Schneider ${ }^{18}$ for estimating the maximal anaerobic contribution in non-athlete individuals. Participants remained quietly on the cycle ergometer for $5 \mathrm{~min}$ to determine $\mathrm{O}_{2}$ baseline. Then, the participants warmed up for $4 \mathrm{~min}$ at $30 \mathrm{~W}$. After that, the power output was adjusted to $110 \%$ of PPO. Participants were asked to maintain pedal cadence between $60-70 \mathrm{rpm}$. The test was finished when the pedal cadence was less than $60 \mathrm{rpm}$ for more than five consecutive seconds. Verbal encouragement was used during the test. Peak oxygen uptake was defined as the average of the last $30 \mathrm{~s}$ of the test. Blood samples were collected from the ear lobe at rest and at immediately, 3 and 5 min after the exercise. Peak lactate was defined as the highest value found after the end of the test. The RPE was recorded immediately after the test using the Borg Scale (6-20) ${ }^{19}$.

Net aerobic energy was estimated by subtracting $\mathrm{VO}_{2}$ at rest from the $\mathrm{VO}_{2}$ area integrated over time during test by the trapezoidal method. The contribution of the anaerobic alactic system was considered to be the fast component of excess postexercise oxygen consumption $(10 \mathrm{~min})$. The breath-by-breath $\mathrm{VO}_{2}$ data were fitted to a monoexponential model and anaerobic alactic system was obtained by integration of the exponential part. To estimate anaerobic lactic energy a value of $1 \mathrm{mmol} \mathrm{1}^{-1}$ was considered to be equivalent to $3 \mathrm{ml} \mathrm{O}_{2} \mathrm{~kg}^{-1}$ body mass ${ }^{20}$. A

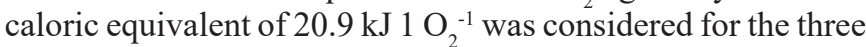
energy systems. Total energy expenditure was calculated as the sum of the energy expenditure of the three energy systems. All calculations were made using the free software GEDAE-LaB ${ }^{\circledR 21}$, available at http://www.gedaelab.org.

\section{CHO mouth rinse}

Participants washed their mouth immediately before the trial with a solution containing $25 \mathrm{ml}$ of $6.4 \%$ of maltodextrin $(\mathrm{CHO})$ or PLA (juice without CHO). The solutions had the same taste, smell and texture. Participants were asked to perform tongue movements to keep the solution in the mouth during $10 \mathrm{~s}$ and then spat the fluid out into a bowl, as recommended ${ }^{22}$.

\section{Statistical analysis}

The data distribution was analyzed using the Shapiro-Wilk test. Data are reported as mean and standard deviation (SD). A paired t test was used to examine the differences between $\mathrm{CHO}$ and PLA conditions for time to exhaustion, and aerobic, anaerobic lactic and anaerobic alactic contributions, and lactate peak. The Wilcoxon test was used to compare the $\mathrm{CHO}$ and 
PLA groups for the variables that did not present normality in the distribution, such as the total contribution of the energy systems and the RPE. The level of significance was set at $p$ $<0.05$. All statistical calculations were performed with SPSS 17.0 for Windows (SPSS, Inc., Chicago, IL).

The effect size was calculated for all carbohydrate and PLA variables. The thresholds for the small, moderate, and large effects were $0.20,0.50$, and 0.80 , respectively. The effect size was determined by the formula: (mean1 - mean2) / grouped standard deviation ${ }^{23}$.

\section{Results}

Time to exhaustion was similar between $\mathrm{CHO}$ and PLA conditions (CHO: $174.3 \pm 42.8 \mathrm{~s}$; PLA: $166.7 \pm 26.3 \mathrm{~s} ; \mathrm{p}=0.33$; effect size $=0.21)$. However, the RPE at the exhaustion reduced with CHO mouth rinse $(p=0.02$; effect size $=0.84$; Fig 1$)$.

Figure 1. Rating of perceived exertion (RPE) at the moment of exhaustion with $\mathrm{CHO}$ and PLA mouth rinse.

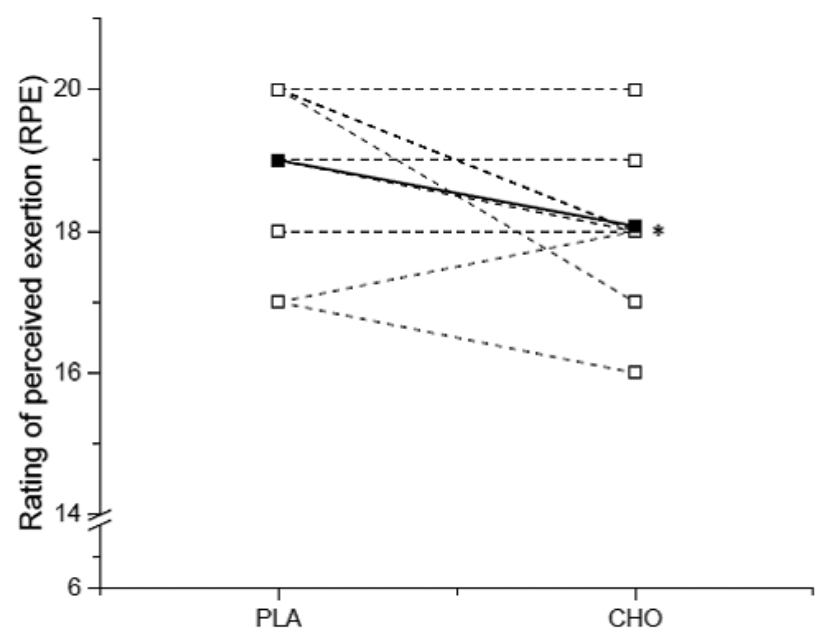

There was no significant difference for aerobic $(\mathrm{p}=0.34$; effect size $=0.13)$, anaerobic lactic $(p=0.10$; effect size $=0.26)$, and anaerobic alactic $(\mathrm{p}=0.37$; effect size $=0.31)$ contribution as well as total energy expenditure $(p=0.15$; effect size $=0.31)$ (Table 1). There was also no difference in lactate peak between $\mathrm{CHO}$ and PLA conditions (13.4 \pm 2.8 and $12.5 \pm 2.3 \mathrm{mmol}, \mathrm{p}$ $=0.12$; effect size $=0.31$, respectively).

\section{Discussion}

To the best of our knowledge, this is the first study investigating the effect of pre-exercise mouth rinse on energy systems contribution during a high-intensity exercise. The results of the present study provide interesting new insights considering the effects of the $\mathrm{CHO}$ mouth rinse, which suggests that $\mathrm{CHO}$ mouth rinse does neither improve time to exhaustion nor alter energy systems contribution during a high-intensity exercise in physically active man, but attenuated RPE at the exhaustion.

Several studies showed that rinsing the mouth with $\mathrm{CHO}$ improves the performance during high-intensity exercises ${ }^{5,6,7}$. However, in the present study, we were unable to find an ergogenic effect of $\mathrm{CHO}$ mouth rinse during a high-intensity exercise performed at $110 \%$ of PPO. The differences concerning the exercise and mouth rinse protocols between our and these studies may have generated this divergence. For example, Beaven, Maulder, Pooley, KilduffL, $\mathrm{Cook}^{5}$ investigated $\mathrm{CHO}$ mouth rinse effects on multiple sprints $(5 \times$ x 6 -s sprint with $24 \mathrm{~s}$ recovery between them), while Phillips, Findlay S, Kavaliauskas M, Grant ${ }^{7}$ and Chong, Guelfi, Fournier ${ }^{6}$ investigated $\mathrm{CHO}$ mouth rinse effect on a 30 -s and 45-s all-out exercise, respectively. In all of these studies, performance was improved with $\mathrm{CHO}$ mouth rinse. Another possibility would be the number of mouth rinses. In these studies ${ }^{6,7}$ the participants performed a greater number of oral rinses before the test ( $\sim 9$ mouthwashes). However, Beaven, Maulder, Pooley, Kilduff, $\mathrm{Cook}^{5}$ used only one rinse before a 6 -s sprint and found that $\mathrm{CHO}$ mouth rinse rapidly enhanced the sprint power in the first, thus requiring more studies elucidating the effects of multiple oral rinses compared to just one previous oral rinse.

Similar to Chong, Guelfi, Fournier ${ }^{6}$ and Phillips, Findlay, Kavaliauskas, Grant ${ }^{7}$, our study did not find difference in the lactatemia. Furthermore, no significant differences for aerobic, anaerobic lactic, anaerobic alactic and total energy expenditure were found in the present study. Regarding the anaerobic contribution in high intensity exercise, studies verifying the effect of caffeine ${ }^{24}$ and metformin ${ }^{25}$ showed that even with a higher time to exhaustion, there was no significant difference in the anaerobic contribution in these exercises, probably because the anaerobic energy reserve may be a finite source ${ }^{26}$. On the other hand, as well as $\mathrm{CHO}$ mouth rinse, $\mathrm{CHO}$ intake did not increase time to exhaustion and anaerobic contribution in physically active cycling at $110 \% \mathrm{PPO}^{27}$.

Nevertheless, even without significant difference, time to exhaustion was $4.4 \%$ longer in $\mathrm{CHO}$ than in PLA, and was

Table 1. Energy systems contribution in CHO and PLA conditions.

\begin{tabular}{ccccc}
\hline & $\begin{array}{c}\text { CHO } \\
\text { Contribution (kJ) }\end{array}$ & $\begin{array}{c}\text { PLA Contribution } \\
(\mathbf{k J})\end{array}$ & $\begin{array}{c}\text { CHO } \\
\text { Contribution (\%) }\end{array}$ & $\begin{array}{c}\text { PLA Contribution } \\
(\%)\end{array}$ \\
\hline $\mathbf{W}_{\text {AER }}$ & $135.1 \pm 41.2$ & $129.8 \pm 35.3$ & 66.4 & 66.1 \\
$\mathbf{W}_{\text {PCR }}$ & $57.6 \pm 17.1$ & $53.4 \pm 15.1$ & 28.3 & 27.2 \\
$\mathbf{W}_{\text {[La] }}$ & $10.4 \pm 8.4$ & $13.2 \pm 9.2$ & 5.3 & 6.7 \\
$\mathbf{W}_{\text {TOTAL }}$ & $203.2 \pm 46.4$ & $196.5 \pm 45.2$ & 100 & 100 \\
\hline
\end{tabular}

$\mathrm{W}_{\mathrm{AER}}$ - aerobic contribution. $\mathrm{W}_{\mathrm{PCR}}$ - anaerobic alactic contribution. $\mathrm{W}_{[\mathrm{La}]}$ - anaerobic lactic contribution. $\mathrm{W}_{\mathrm{TOTAL}}$ - total energy expenditure. PLA - placebo. CHO carbohydrate. 
associated with a lower RPE, possibly because $\mathrm{CHO}$ mouth rinse may have activated brain regions (including areas of the insula/frontal operculum, orbitofrontal cortex and striatum) involved in reward ${ }^{8,9}$. This result corroborates recent findings ${ }^{28}$ showing that even with a reduced RPE at the exhaustion after CHO mouth rinse, exercise endurance at $110 \%$ of PPO was not altered after a $\mathrm{CHO}$ mouth rinse. It was showed that $\mathrm{CHO}$ rinsing is able to maintain neuromuscular activation (i.e., the electromyographic signal of the vastus lateralis) along of time during moderate-, but not high-intensity exercise ${ }^{28}$. Probably, an activation of reward areas with $\mathrm{CHO}$ rinse is able to reduce feelings of fatigue during the high-intensity exercise, but it is not sufficient to translate in an improvement in the exercise performance in this kind of exercise.

A potential limitation of our study was to have used a blind model rather than double blind. However, to minimize this limitation, verbal encouragement was standardized for all tests.

\section{Conclusions}

The present study showed that $\mathrm{CHO}$ mouth rinse did not increase time to exhaustion or alter energy systems contribution during a high-intensity exercise in physically active man. However, this seems to attenuate RPE at the exhaustion.

\section{References}

1. Coyle EF, Coggan AR, Hemmert MK, Ivy JL. Muscle glycogen utilization during prolonged strenuous exercise when fed carbohydrate. J Appl Physiol (1985). 1986;61(1):165-72.

2. Newell ML, Hunter AM, Lawrence C, Tipton KD, Galloway S DR. The Ingestion of 39 or $64 \mathrm{~g} \cdot \mathrm{h}(-1)$ of Carbohydrate is Equally Effective at Improving Endurance Exercise Performance in Cyclists. Int J Sport Nutr Exerc Metab. 2015;25(3):285-92. doi: 10.1123/ijsnem.2014-0134.

3. Bergström J, Hultman E. A study of the glycogen metabolism during exercise in man. Scand J Clin Lab Invest. 1967;19(3):218-28.

4. Pottier A, Bouckaert J, Gilis W, Roels T, Derave W. Mouth rinse but not ingestion of a carbohydrate solution improves 1-h cycle time trial performance. Scand J Med Sci Sports. 2010;20(1):10511. doi: 10.1111/j.1600-0838.2008.00868.x.

5. Beaven CM, Maulder P, Pooley A, Kilduff L, Cook C. Effects of caffeine and carbohydrate mouth rinses on repeated sprint performance. Appl Physiol Nutr Metab. 2013;38(6):633-7. doi: 10.1139/apnm-2012-0333.

6. Chong E, Guelfi KJ, Fournier PA. Combined glucose ingestion and mouth rinsing improves sprint cycling performance. Int J Sport Nutr Exerc Metab. 2014;24(6):605-12. doi: 10.1123/ ijsnem.2013-0097.

7. Phillips SM, Findlay S, Kavaliauskas M, Grant MC. The Influence of Serial Carbohydrate Mouth Rinsing on Power Output during a Cycle Sprint. J Sports Sci Med. 2014;13(2):252-8.

8. Chambers ES, Bridge MW, Jones DA. Carbohydrate sensing in the human mouth: effects on exercise performance and brain activity. J Physiol. 2009;587(8):1779-94. doi: 10.1113/ jphysiol.2008.164285.
9. Turner CE, Byblow WD, Stinear CM, Gant N. Carbohydrate in the mouth enhances activation of brain circuitry involved in motor performance and sensory perception. Appetite. 2014;80:212-9. doi: 10.1016/j.appet.2014.05.020.

10. Gant N, Stinear CM, Byblow WD. Carbohydrate in the mouth immediately facilitates motor output. Brain Res. 2010;1350:151-8. doi: 10.1016/j.brainres.2010.04.004.

11. Carter JM, Jeukendrup AE, Jones DA. The effect of carbohydrate mouth rinse on 1-h cycle time trial performance. Med Sci Sports Exerc. 2004;36(12):2107-11.

12. Fraga C, Velasques B, Koch AJ, Machado M, Paulucio D, Ribeiro P, et al. Carbohydrate mouth rinse enhances time to exhaustion during treadmill exercise. Clin Physiol Funct Imaging. 2017;37(1):17-22. doi: 10.1111/cpf.12261.

13. Stølen T, Chamari K, Castagna C, Wisløff U. Physiology of soccer: an update. Sports Med. 2005;35(6):501-36.

14. Lane SC, Bird SR, Burke LM, Hawley JA. Effect of a carbohydrate mouth rinse on simulated cycling time-trial performance commenced in a fed or fasted state. Appl Physiol Nutr Metab. 2013;38(2):134-9. doi: 10.1139/apnm-2012-0300.

15. Jackson AS, Pollock ML. Practical assessment of body composition. Physician Sportsmed. 1985;13:76-90.

16. Siri WE. Body composition from fluids spaces and density: analyses of methods. In: Techniques for measuring body composition, Washington, DC: National Academy of Science and Natural Resource Council, 1961.

17. Howley ET, Bassett DR Jr, Welch HG. Criteria for maximal oxygen uptake: review and commentary. Med Sci Sports Exerc. 1995;27(9):1292-301.

18. Weber CL, Schneider DA. Reliability of MAOD measured at $110 \%$ and $120 \%$ of peak oxygen uptake for cycling. Med Sci Sports Exerc. 2001;33(6):1056-9.

19. Borg GA. Psychophysical bases of perceived exertion. Med Sci Sports Exerc. 1982;14:377-81.

20. di Prampero PE, Ferretti G. The energetics of anaerobic muscle metabolism: a reappraisal of older and recent concepts. Respir Physiol. 1999;118(2-3):103-15.

21. Bertuzzi R, Melegati J, Bueno S, Ghiarone T, Pasqua LA, Gáspari AF, et al. GEDAE-LaB: A Free Software to Calculate the Energy System Contributions during Exercise. PLoS One. 2016;11(1):e0145733. doi: 10.1371/journal.pone.0145733.

22. Sinclair J, Bottoms L, Flynn C, Bradley E, Alexander G, McCullagh S, et al. The effect of different durations of carbohydrate mouth rinse on cycling performance. Eur J Sport Sci. 2014;14(3):259-64. doi: 10.1080/17461391.2013.785599.

23. Cohen J. Statistical power analysis for the behavioral sciences. $2^{\mathrm{a}}$ ed. Hillsdale: Erlbaum, 1988.

24. Poli RA, Miyagi WE, Nakamura FY, Zagatto AM. Caffeine Improved Time to Exhaustion But Did Not Change Alternative Maximal Accumulated Oxygen Deficit Estimated During a Single Supramaximal Running Bout. Int J Sport Nutr Exerc Metab. 2016;26(6):549-557. doi: 10.1123/ijsnem.2016-0038.

25. Learsi SK, Bastos-Silva VJ, Lima-Silva AE, Bertuzzi R, De Araujo GG. Metformin improves performance in highintensity exercise, but not anaerobic capacity in healthy male subjects. Clin Exp Pharmacol Physiol. 2015;42(10):1025-9. doi: 10.1111/1440-1681.12474. 
26. Margaria R, Aghemo P, Rovelli E. Measurement of muscular power (anaerobic) in man. J Appl Physiol. 1966;21(5):1662-4.

27. Bastos-Silva VJ, Learsi SK, Melo AA, Lima-Silva AE, De Araujo GG. Effects of carbohydrate intake on time to exhaustion and anaerobic contribution during supramaximal exercise. Rev Nutr. 2016;29:691-697. doi: 10.1590/1678-98652016000500007.

28. Bastos-Silva VJ, Melo Ade A, Lima-Silva AE, Moura FA, Bertuzzi R, de Araujo GG. Carbohydrate Mouth Rinse Maintains Muscle Electromyographic Activity and Increases Time to Exhaustion during Moderate but not High-Intensity Cycling Exercise. Nutrients. 2016;8(3):49. doi: 10.3390/nu8030049.

\section{Acknowledgments}

The authors thank the Laboratory of Physical Fitness, Performance and Health (LAFIDES) of the Federal University of Alagoas. The authors thank The Coordination for the Improvement of Higher Education Personnel (CAPES), The Research
Alagoas Foundation (FAPEAL), and National Council for Scientific and Technological Development $(\mathrm{CNPq})$ by financial support.

\section{Corresponding author}

Gustavo Gomes de Araujo

Full postal address: Campus A.C. Simões; Av. Lourival Melo Mota, S/N, Tabuleiro do Martins, 57.072-970 - Maceió, AL, Brazil.

Email: gusta_ef@yahoo.com.br

Manuscript received on June 28, 2016

Manuscript accepted on August 24, 2016

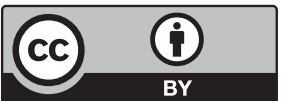

Motriz. The Journal of Physical Education. UNESP. Rio Claro, SP, Brazil - eISSN: 1980-6574 - under a license Creative Commons - Version 3.0 